\title{
The Basal Forebrain Cholinergic System Is Required Specifically for Behaviorally Mediated Cortical Map Plasticity
}

\author{
Dhakshin Ramanathan, ${ }^{1}$ Mark H. Tuszynski, ${ }^{1,2}$ and James M. Conner ${ }^{1}$ \\ ${ }^{1}$ Department of Neurosciences, University of California, San Diego, La Jolla, California 92093-0626, and 2Veterans Affairs Medical Center, San Diego, \\ California 92161
}

The basal forebrain cholinergic system has been implicated in the reorganization of adult cortical sensory and motor representations under many, but not all, experimental conditions. It is still not fully understood which types of plasticity require the cholinergic system and which do not. In this study, we examine the hypothesis that the basal forebrain cholinergic system is required for eliciting plasticity associated with complex cognitive processing (e.g., behavioral experiences that drive cortical reorganization) but is not required for plasticity mediated under behaviorally independent conditions. We used established experimental manipulations to elicit two distinct forms of plasticity within the motor cortex: facial nerve transections evoke reorganization of cortical motor representations independent of behavioral experience, and skilled forelimb training induces behaviorally dependent expansion of forelimb motor representations. In animals that underwent skilled forelimb training in conjunction with a facial nerve lesion, cholinergic mechanisms were required for mediating the behaviorally dependent plasticity associated with the skilled motor training but were not necessary for mediating plasticity associated with the facial nerve transection. These results dissociate the contribution of cholinergic mechanisms to distinct forms of cortical plasticity and support the hypothesis that the forebrain cholinergic system is selectively required for modulating complex forms of cortical plasticity driven by behavioral experience.

\section{Introduction}

Extensive evidence has implicated the basal forebrain cholinergic system in regulating cortical plasticity. Pairing a sensory event with a cholinergic input selectively induces cortical plasticity associated with the paired stimulus (Kilgard and Merzenich, 1998; Weinberger, 2003; Ma and Suga, 2005). Likewise, lesions of the basal forebrain cholinergic system prevent cortical plasticity from occurring in many experimental paradigms (Juliano et al., 1991; Webster et al., 1991; Sachdev et al., 1998; Conner et al., 2003; Prakash et al., 2004; Conner et al., 2005). Together, these results suggest that acetylcholine is an essential substrate for mediating cortical map plasticity in the adult animal. This theory was challenged by a recent study (Kamke et al., 2005) demonstrating the reorganization of tonotopic representations within auditory cortex after cochlear injury, even after the elimination of cholinergic innervation to that area. Despite some caveats (Kilgard, 2005), this study clearly indicated that the prevailing theory - that the basal forebrain cholinergic system is essential for cortical map plasticity-needed revising.

In the present study, we postulate that the cholinergic system

\footnotetext{
Received Jan. 15, 2009; revised Feb. 23, 2009; accepted March 10, 2009.

This work was supported by the National Institutes of Health, the Veterans Administration, and the Dr. Miriam and Sheldon G. Adelson Medical Research Foundation.

Correspondence should be addressed to either Dr. James M. Conner or Dr. Mark H. Tuszynski, Department of Neurosciences, University of California, San Diego, 9500 Gilman Drive, Mail Code 0626, La Jolla, CA 92023, E-mail: jmconner@ucsd.edu or mtuszynski@ucsd.edu.

DOI:10.1523/JNEUROSCI.0230-09.2009

Copyright $\odot 2009$ Society for Neuroscience $\quad$ 0270-6474/09/295992-09\$15.00/0
}

is essential only for cortical map plasticity driven by behavioral experience [specifically that portion of behavioral training when cortical reorganization takes place (Conner et al., 2003)] and is not essential for plasticity that occurs independent of behavioral activity. In this model, acetylcholine does not gate all forms of plasticity; rather, it plays an essential role in the execution of the selective attention, learning, and memory required for plasticity mediated during behavioral training, a role for acetylcholine already substantiated by a wealth of evidence (Wilson and Rolls, 1990; Richardson and DeLong, 1991; Bakin et al., 1996; Bakin and Weinberger, 1996; Butt et al., 1997; Sarter and Bruno, 1997; McLin et al., 2002; Sarter et al., 2005).

Because the motor cortex exhibits plasticity in both behaviorally dependent and behaviorally independent contexts, it is ideally suited to test this hypothesis. Extensive reorganization of motor cortical representations occurs rapidly and without any behavioral activity following transection of the facial motor nerve (Semba and Egger, 1986; Sanes et al., 1988). Furthermore, the acquisition of a skilled motor behavior, or skilled motor training after an injury, results in a significant expansion of the cortical motor representation for the trained body part (Nudo and Milliken, 1996; Nudo et al., 1996b; Kleim et al., 1998; Liepert et al., 1998; Conner et al., 2003; Nelles, 2004; Conner et al., 2005; Ramanathan et al., 2006). Using these two paradigms, we now assess the role of the basal forebrain cholinergic system in mediating both behaviorally dependent and behaviorally independent forms of cortical motor map plasticity.

In this study, we first investigate the effects of cholinergic- 


\section{Experiment 1}

SAP or ACSF
Injection

\section{Experiment 2}

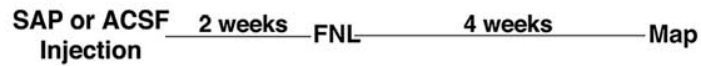

\section{Experiment 3}

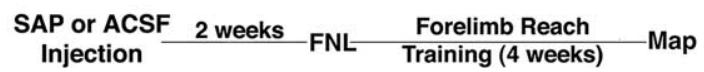

Figure 1. Experimental paradigm. Three experiments were conducted in this study. Experiment 1, An initial experiment (10 rats) determined whether cholinergic mechanisms were required for mediating plasticity of cortical motor representations acutely following facial nerve transections (FNL), whereby plasticity is induced rapidly (within minutes to hours) and occurs independent of behavioral activity. Experiment 2, A second experiment (23 rats) examined whether the long-term maintenance of cortical plasticity following a facial nerve transection is dependent on cholinergic mechanisms. Experiment 3, A final experiment (32 rats) examined the effects of selective cholinergic depletion on cortical plasticity mediated concurrently by nonbehavioral (facial nerve transection) and behavioral (skilled motor training) paradigms within the same animal. See text for detailed methods use in each experiment.

specific lesions of the basal forebrain on the induction and maintenance of behaviorally independent cortical motor map plasticity after transection of the facial motor nerve. Next, we examine the effects of cholinergic depletion in animals with a facial nerve transection that also underwent skilled motor training. Thus, the requirement of cholinergic inputs was concurrently assessed for both behaviorally and nonbehaviorally mediated cortical reorganization, within the same cortical domain and in the same animal, allowing us to test the hypothesis that cholinergic inputs are specifically required for cortical map plasticity associated with behavioral experience.

\section{Materials and Methods}

Experimental paradigm. A total of 65 adult Fischer 344 rats (following exclusion of 6 animals described below) were examined in three separate experiments (Fig. 1). To examine the role of basal forebrain cholinergic mechanisms in each paradigm, animals underwent either bilateral cholinergic depletion using the 192-IgG-saporin (SAP) immunotoxin (Advanced Targeting) or received similar control injections of artificial cerebral spinal fluid (ACSF). In all cases, the extent of cholinergic depletion was quantified by assessing the degree of loss of acetylcholinesterase (AChE)-positive afferent terminals innervating the motor cortex. Only animals with $>95 \%$ depletion of cholinergic fibers were included for additional analysis. The specificity of the immunotoxin for the cholinergic component of the basal forebrain was also confirmed by assessing potential loss of GABAergic components of the basal forebrain, as described previously (Heckers et al., 1994; Baxter et al., 1995; Pang et al., 2001; Conner et al., 2003).

Experiment 1 (Fig. 1) was designed to specifically examine cortical map reorganization occurring independent of behavioral activity. For this purpose, we used an established paradigm in which plasticity is rapidly induced within the motor cortex after facial motor nerve transection. For this portion of the study, 10 animals initially received injections of either SAP $(n=5)$ or ACSF $(n=5)$ into the basal forebrain. Six weeks after these injections, acute plasticity induced by a facial nerve lesion was assessed in a single surgical session by comparing pretransection and posttransection maps from within the same animal. To achieve this goal, animals were anesthetized and mapped bilaterally using a modified intracortical microstimulation (ICMS) technique (see below). Immediately after the initial mapping procedure, while animals remained anesthetized in the stereotaxic frame, the facial motor nerve was transected bilaterally. Two hours after the transection, animals were remapped to examine acute cortical map plasticity. These postlesion maps were com- pared with the prelesion maps in both SAP-injected and vehicle-injected animals using paired $t$ tests.

Experiment 2 (Fig. 1) examined the long-term maintenance of plasticity in the motor cortex imposed by a facial motor nerve transection. In this experiment, 28 animals initially received SAP $(n=13)$ or $\operatorname{ACSF}(n=$ 15) injections. Two weeks later, all animals underwent bilateral facial motor nerve transections. Intracortical mapping was then carried out 4 weeks after the facial motor nerve transaction using standard ICMS techniques (see below). To assess plasticity after long-term facial nerve lesion, maps derived from these animals were compared with the acute prelesion and postlesion maps from animals in experiment 1 , using multivariate ANOVA. Five animals were excluded from this portion of the study because of incomplete cholinergic lesions ( $<95 \%$ cholinergic depletion).

Experiment 3 (Fig. 1) examined the effects of cholinergic lesions on behaviorally dependent and behaviorally independent forms of cortical plasticity induced simultaneously within the motor cortex. In this experiment, 33 animals initially received either SAP $(n=16)$ or ACSF $(n=17)$ injections into the basal forebrain. Two weeks later, all animals were given bilateral facial motor nerve transections. One week after the facial nerve lesions, one-half of the animals began a 3-week regimen of skilled motor training, while the remaining animals served as untrained (caged) controls. At the completion of the 3-week training period, all animals were mapped using ICMS techniques (see below). Multivariate ANOVA was used to compare mapping data obtained from SAP-injected and vehicle-injected animals within this portion of the study, along with mapping data obtained from experiments 1 and 2. In this way, it was possible to determine the requirement for cholinergic mechanisms in mediating both behaviorally dependent and independent forms of cortical map plasticity. One animal was excluded from experiment 3 because of an incomplete cholinergic lesion.

Selective cholinergic depletion. Selective ablation of the basal forebrain cholinergic system was carried out according to previously described procedures (Conner et al., 2003). The immunotoxin 192-IgG-SAP (Advanced Targeting Systems), diluted to a concentration of $0.375 \mathrm{mg} / \mathrm{ml}$ in ACSF, was injected bilaterally into the nucleus basalis magnocellularis/ substantia inominata using a $1.0 \mu \mathrm{l}$ Hamilton syringe. The following sites and volumes were injected: site $1(0.3 \mu \mathrm{l}$ each side $), \mathrm{R} / \mathrm{C}=-1.4 \mathrm{~mm}$, $\mathrm{M} / \mathrm{L}= \pm 2.5 \mathrm{~mm}, \mathrm{D} / \mathrm{V}=-8.0 \mathrm{~mm}$; site $2(0.2 \mu$ l each side $), \mathrm{R} / \mathrm{C}=-2.6$ $\mathrm{mm}, \mathrm{M} / \mathrm{L}= \pm 4.0 \mathrm{~mm}, \mathrm{D} / \mathrm{V}=-7.0 \mathrm{~mm}$. Control animals were similarly injected with vehicle (ACSF) alone.

Facial motor nerve transactions. Facial motor nerve transections were carried out using previously described techniques (Semba and Egger, 1986; Sanes et al., 1988). After anesthesia, skin incisions were made approximately two-thirds of the distance from the ear to the eye. The facial motor nerve was dissected from fascia as it emerged from the parotid gland. Three branches of the facial nerve were transected (the buccal, marginal mandibular, and zygomatic branches) to ensure elimination of whisker movements. A $2 \mathrm{~mm}$ section of each nerve was removed to ensure that peripheral nerve regeneration would not occur within the time span of these experiments.

Functional ICMS mapping. In most cases, standard microelectrode stimulation techniques were used to derive maps of the motor cortex (Kleim et al., 1998; Conner et al., 2003). Animals were anesthetized with ketamine hydrochloride (70 mg/kg i.p.) and xylazine ( $5 \mathrm{mg} / \mathrm{kg}$ i.p.) and received supplemental doses of the anesthesia mixture as needed. Pulledglass-stimulating electrodes (input impedance $\sim 0.5 \mathrm{MOhm}$ at $300 \mathrm{~Hz}$ ) filled with $3 \mathrm{M} \mathrm{NaCl}$ were used. Microelectrode penetrations were made at $500 \mu \mathrm{m}$ intervals at a depth of $\sim 1700 \mu \mathrm{m}$ (corresponding to cortical layers V-VI). Stimulation consisted of a $30 \mathrm{~ms}$ train of $200 \mu$ s duration monophasic cathodal pulses delivered at $333 \mathrm{~Hz}$ from an electrically isolated, constant-current stimulator (AMPI Isoflex) under the control of a programmable pulse generator (AMPI Master-8). Two pulse trains were delivered $1.2 \mathrm{~s}$ apart, and evoked movements were examined with the animal maintained in a prone position and the limbs supported in a consistent manner. At each site, the current was gradually increased until a movement was detected (threshold current), and the movement type was recorded. If no movement was detected at $200 \mu \mathrm{A}$, the site was defined as "nonresponsive."

A modification to the standard ICMS protocol was used in experiment 
1 to investigate acute changes in plasticity mediated by facial nerve transection. This modification was used to control for a potential caveat in interpreting ICMS-derived data following peripheral nerve lesions. Normally, using standard ICMS techniques, only movements evoked by the minimum stimulation threshold are recorded (Nudo et al., 1996b; Kleim et al., 1998; VandenBerg et al., 2002; Conner et al., 2003). After a facial nerve transaction, however, cortically evoked vibrissa movements are no longer possible at any stimulus intensity. Thus, simply increasing the stimulation current may evoke nonvibrissal movements, either by unmasking other movements represented in the same cortical area or by enabling additional current spread and evoking movements from adjacent cortical areas. Map changes arising from either of these two scenarios do not require electrophysiological changes to take place and do not represent genuine plasticity. To control for both of these possibilities when examining acute map plasticity after a facial nerve transection, we modified the routine ICMS mapping procedure. During prefacial nerve lesion mapping, stimulation currents at all vibrissal sites were increased to the maximum ICMS stimulus intensity $(200 \mu \mathrm{A})$ to identify any secondary nonvibrissal movements that could be evoked (see Fig. $3 A, B$ ). Vibrissal sites at which neck or forelimb movements were elicited at higher stimulation currents were then included in the calculation of the prelesion area of neck and forelimb representations (see Fig. 3B). Use of this modification permits identification of genuine shifts in the somatotopic maps (plasticity) as a consequence of the facial nerve lesion. In all cases, the area of the forelimb and neck representations was determined by multiplying the number of responsive sites evoking a movement of the forelimb or neck (including those that existed at higher thresholds within the vibrissal area of untransected animals), respectively, by $0.25 \mathrm{~mm}^{2}$ (i.e., the distance between electrode penetration sites was $0.5 \mathrm{~mm}$ ).

Skilled motor training. Motor training was carried out using singlepellet retrieval boxes as described in detail elsewhere (Conner et al., 2003, 2005). Rats were tested for a total of $12 \mathrm{~d}$, during which total reaches, accuracy, and limb use was recorded. A "reach" was scored when the rat extended its forelimb through the slot. A "hit" was scored if the rat successfully brought the pellet back to his mouth and consumed it. Testing order was randomized daily.

Histology. After electrophysiological mapping, rats were perfused with $75 \mathrm{ml}$ of phosphate buffered saline and $250 \mathrm{ml}$ of $4 \%$ paraformaldehyde in $0.1 \mathrm{M}$ phosphate buffer. Coronal sections $(40 \mu \mathrm{m})$ were cut on a sliding microtome, and a series of sections, spaced $240 \mu \mathrm{m}$ apart, were processed for [AChE histochemistry using the modified Tago method (Di Patre et al., 1993)]. Free-floating sections were washed briefly in $0.05 \mathrm{M}$ Trismaleate buffer, $\mathrm{pH} 5.7$, incubated for $10 \mathrm{~min}$ in Tris-maleate buffer containing $6 \mu \mathrm{g} / \mathrm{ml}$ promethazine, and washed two additional times in Trismaleate buffer. Sections were incubated for $30 \mathrm{~min}$ in a $32.5 \mathrm{~mm}$ Trismaleate buffer solution containing $5 \mathrm{~mm}$ sodium citrate, $3 \mathrm{~mm}$ cupric sulfate, $0.5 \mathrm{~mm}$ potassium ferrocynide, and $0.52 \mathrm{mg} / \mathrm{ml}$ acetylthiocholine iodide, then rinsed five times in $50 \mathrm{~mm}$ Tris- $\mathrm{HCl}, \mathrm{pH}$ 7.6. Sections were incubated for $5 \mathrm{~min}$ in $50 \mathrm{~mm}$ Tris- $\mathrm{HCl}$ containing $0.25 \mathrm{mg} / \mathrm{ml}$ diaminobenzidine tetrahydrochloride and $3 \mathrm{mg} / \mathrm{ml}$ nickel ammonium sulfate. Hydrogen peroxide $(0.006 \%$ final concentration) was added, and sections were allowed to incubate for 2-3 more minutes. The reaction was stopped by washing sections three to four times in $50 \mathrm{~mm}$ Tris- $\mathrm{HCl}$ buffer. Sections were mounted on to gel-subbed slides, dehydrated in a graded series of alcohols, cleared, and coverslipped.

Unbiased sampling techniques were used to estimate the degree of loss in cholinergic innervation to the sensorimotor cortex after SAP injections (Geula and Mesulam, 1996; Conner et al., 2001). The density of cholinergic innervation to the sensorimotor cortex was estimated by counting the number of AChE-positive fibers crossing the two inclusion boundaries of a $15 \mu \mathrm{m}$ counting frame. A stereology computer program (StereoInvestigator) controlled placement of the counting frame within a proscribed sampling area that included cortical layers II-III of sensorimotor cortex (Zilles, 1985). The distance between sampling sites was 150 $\mu \mathrm{m}$. The average area sampled per animal was $1.67 \pm 0.08 \mathrm{~mm}^{2}$. Previous studies have indicated that SAP lesions result in $>98 \%$ loss in cholinergic innervation to the sensorimotor cortex (Conner et al., 2003). Lesions producing a loss in cortical cholinergic innervation of $<90 \%$ were considered incomplete, and these animals were excluded from additional analysis.

The specificity of the 192-IgG-immunotoxin for cholinergic neurons of the basal forebrain was evaluated using previously reported criteria, wherein SAP lesions should not deplete parvalbumin-positive GABAergic cells within the basal forebrain (Heckers et al., 1994; Baxter et al., 1995; Pang et al., 2001; Conner et al., 2003). Parvalbumin-positive GABAergic neurons were examined in tissue sections from both SAP and vehicle-injected animals using immunohistochemical techniques (MAB1572, Millipore Bioscience Research Reagents used at 1:10,000 dilution).

\section{Results}

Cortical plasticity is rapidly induced following facial nerve transection and is independent of cholinergic mechanisms

Initially, ICMS mapping techniques were used: 1) to characterize the plasticity of cortical motor representations within the first $2 \mathrm{~h}$ after a facial nerve transection and 2) to determine whether cholinergic mechanisms are required to enable cortical plasticity in this paradigm, which is devoid of behavioral activity. Based on previous studies (Sanes et al., 1988, 1990), we postulated that within $2 \mathrm{~h}$ of a facial nerve transection, a loss of cortically excitable sites associated with vibrissal movements would occur, together with an increase in the number of sites capable of evoking eye, neck, or forelimb movements. In addition, we predicted a reduction in stimulation thresholds for evoking these new movements (Sanes et al., 1988, 1990).

Cortical cholinergic innervation was selectively eliminated in 5 of the 10 animals by injecting the cholinergic-specific toxin 192-IgG-SAP bilaterally into the nucleus basalis (Book et al., 1992; Conner et al., 2003, 2005); control animals received injections of ACSF. SAP injections reduced cholinergic innervation to the sensorimotor cortex by $98 \pm 2 \%$ relative to ACSF-injected subjects $(p<0.0001)$ (Fig. 2$)$, consistent with previous reports (Conner et al., 2003, 2005), but resulted in no detectable depletion of GABAergic neurons in the same region (Fig. $2 E, F$ ).

Six weeks after SAP or ACSF injections, cortical plasticity following facial nerve transections was assessed using a modified ICMS mapping approach, as described in detail in Materials and Methods. Initial motor maps obtained before the facial nerve transection indicated that ACSF-injected and SAP-injected animals had comparable neck representations (Fig. 3) (area in ACSF-injected subjects $=1.0 \pm 0.3 \mathrm{~mm}^{2}$; SAP-injected subjects $=0.9 \pm 0.2 \mathrm{~mm}^{2} ; p=0.8$; unpaired $t$ test). Two hours after a facial nerve transection, the size of the neck representation increased significantly in both ACSF-injected (to $1.6 \pm 0.2 \mathrm{~mm}^{2}$ ) and SAP-injected (to $1.9 \pm 0.3 \mathrm{~mm}^{2}$ ) animals compared with their respective pretransection baselines (Fig. $3 ; p<0.01$ for both vehicle and SAP groups compared with pretransection maps, paired $t$ test). The size of the neck representation following a facial nerve transection did not differ significantly in ACSFinjected and SAP-injected groups ( $p=0.2$; unpaired $t$ test), indicating that cholinergic mechanisms were not required for this aspect of plasticity. No significant change in the size of the forelimb representation occurred in either SAP-injected or ACSFinjected animals following a facial nerve transection ( $p=0.7$ for both groups compared with pretransection baseline; paired $t$ test) (Fig. 3).

Mean stimulation thresholds to evoke neck movements decreased by $12 \pm 6 \mu \mathrm{A}$ following facial nerve transection relative to threshold levels before transection ( $p<0.05$; one-sided paired $t$ test), consistent with previous studies (Donoghue et al., 1990). However, stimulation thresholds did not differ significantly comparing SAP-injected and ACSF-injected subjects posttransection ( $p=0.3$; unpaired $t$ test), again suggesting that cholinergic 


\section{Control}
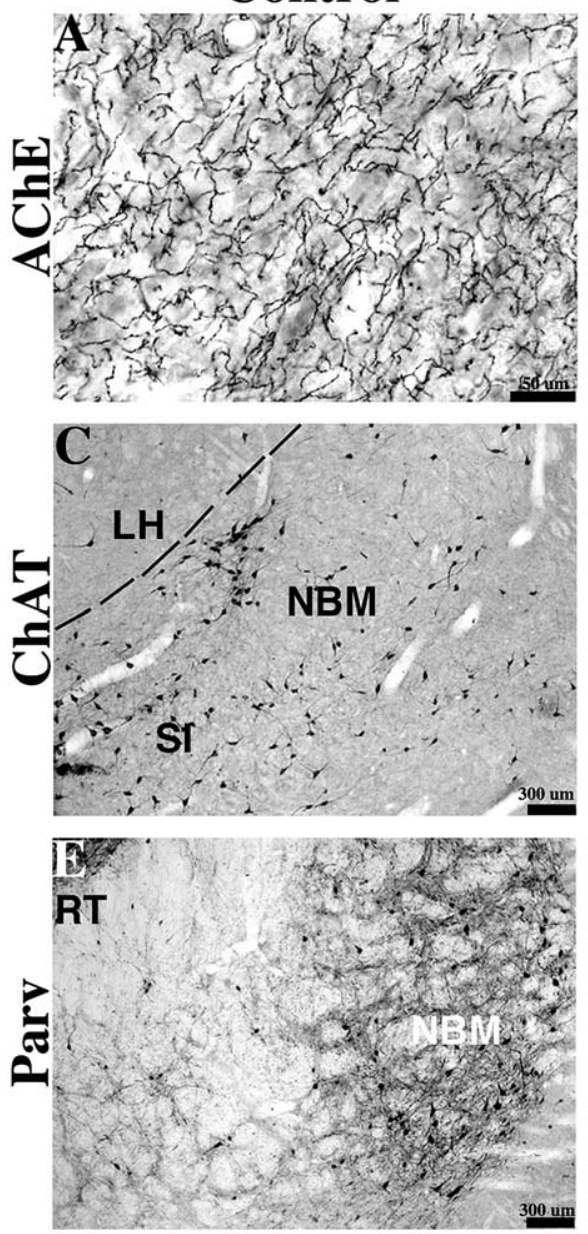

SAP
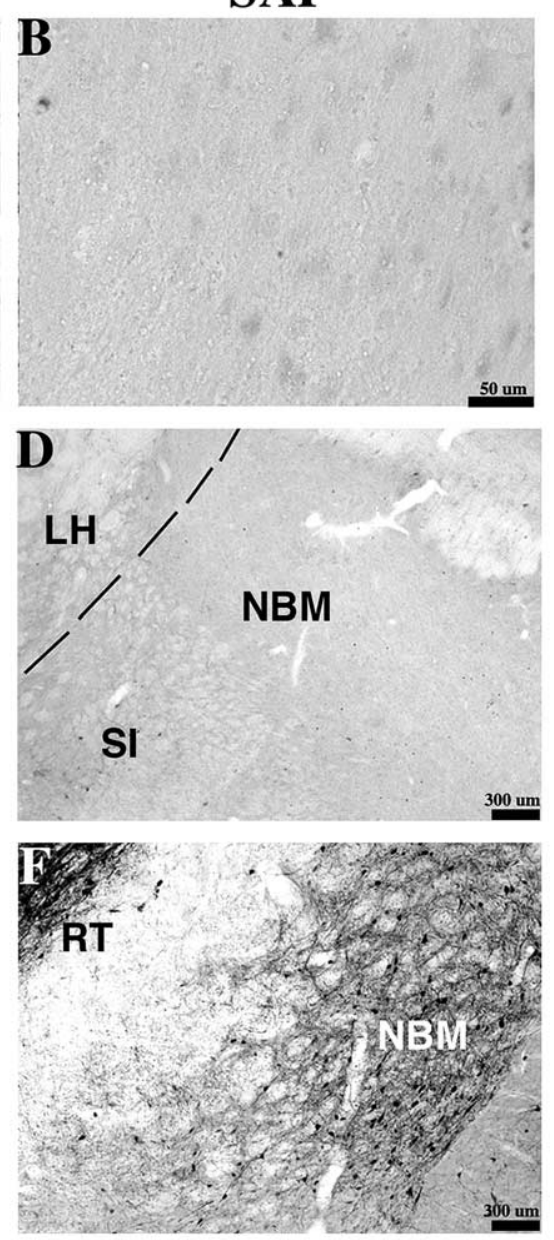

Figure 2. Injection of $192 \mathrm{lg}$ G-saporin into the nucleus basalis selectively eliminates cholinergic innervation to the sensorimotor cortex. $\boldsymbol{A}$, Normal acetylcholinesterase cholinergic fiber histochemistry within layer $2 / 3$ of motor cortex. $\boldsymbol{B}$, Following injections of the cholinergic-specific immunotoxin 192-lgG-saporin into the nucleus basalis and substantia inominata, there is almost a complete loss of afferent cholinergic innervation to the cortex. Within the nucleus basalis and substantia inominata, 192-IgG SAP injections eliminate nearly all ChAT-positive cholinergic neurons $(\boldsymbol{D})$ when compared with ACSF-injected controls (C). Immunohistochemical staining for parvalbumin (parv), a marker for basal forebrain GABAergic neurons, indicated nearly identical patterns in animals receiving either $\mathrm{ACSF}(\boldsymbol{E})$ or $\operatorname{SAP}(\boldsymbol{F})$, thus confirming the selectivity of the immunotoxin for the cholinergic component of the basal forebrain.

mechanisms were not required for this aspect of plasticity. Notably, the mean stimulation threshold to evoke forelimb movements did not change following facial nerve transection in either group ( $p=0.8$; one-sided paired $t$ test), suggesting that the change in threshold observed for neck movements was likely a specific consequence of plasticity within neck representations, rather than a nonspecific state change within the brain as a result of surgery, general anesthesia, or other nonspecific effects.

Long-term maintenance of cortical plasticity following facial nerve transection is independent of cholinergic mechanisms Results from the preceding experiment demonstrate that basal forebrain cholinergic mechanisms are not required for the immediate reorganization of motor representations following a facial nerve transaction. Previous studies have indicated that this behaviorally independent form of map plasticity persists for weeks to months following transection of the facial nerve (Donoghue et al., 1990; Sanes et al., 1990). To determine whether distinct neural mechanisms underlie the acute induction of plasticity following facial nerve transection, versus its longterm maintenance, we conducted additional studies examining the persistence and cholinergic dependence of long-term motor map changes following facial nerve transection. For this purpose, 13 animals received SAP injections into the nucleus basalis, and 15 animals received comparable ACSF injections. Two weeks after the injection procedure, animals were given bilateral facial motor nerve lesions and were returned to their home cage. After an additional 4 weeks, animals were mapped bilaterally using standard ICMS techniques. SAP injections in this group of rats resulted in a $97 \pm 1.0 \%$ reduction in cholinergic innervation to the sensorimotor cortex relative to vehicle-injected rats ( $p<0.001$; unpaired $t$ test).

Facial nerve transections resulted in a significant long-term expansion of neck representations in both ACSF-injected and SAP-injected animals compared with representations from control, pretransected animals (Fig. 4; neck map area $=1.7 \pm 0.2 \mathrm{~mm}^{2}$ in ACSF-injected and $1.8 \pm 0.2 \mathrm{~mm}^{2}$ in SAP-injected animals). The size of neck representations in both groups of chronically lesioned animals was comparable to that observed immediately following facial nerve transection $\left(1.9 \pm 0.2 \mathrm{~mm}^{2}\right)$ and was significantly larger than the mean area of neck representations before the nerve transection $\left(0.9 \pm 0.2 \mathrm{~mm}^{2}\right.$; ANOVA $p<0.01$ across all four groups; Tukey-Kramer HSD, $p<$ 0.05 comparing pretransection and all other groups; no significant differences in any other comparisons).

Consistent with observations from acute transection experiments, there was no difference in the size of the caudal forelimb representation 4 weeks after a facial nerve injury when comparing ACSF- and SAP-treated animals (Fig. $4 B$; ANOVA, $p=0.13$ across all groups). Four weeks after facial nerve lesions, the mean size of the forelimb region was $5.1 \pm 0.2 \mathrm{~mm}^{2}$ in ACSF-injected rats and $5.3 \pm 0.2 \mathrm{~mm}^{2}$ in SAP-lesioned rats. The size of the caudal forelimb area in both long-term lesioned groups was also not statistically different from that of pretransected animals $(4.8 \pm 0.2$ $\left.\mathrm{mm}^{2}\right)$ or acutely lesioned animals $\left(4.7 \pm 0.2 \mathrm{~mm}^{2}\right)$.

\section{Cholinergic mechanisms are essential for behaviorally mediated motor cortex plasticity}

The previous experiments demonstrate that motor cortex plasticity following a facial nerve transection, which occurs independent of behavioral experience, does not require cholinergic mechanisms. To further distinguish the contribution of cholinergic mechanisms required for mediating distinct forms of cortical plasticity, an additional experiment was conducted using a paradigm in which behaviorally dependent and behaviorally independent forms of cortical plasticity were concurrently expressed within a single cortical region. Previous studies have in- 
dicated that skilled motor training is associated with the expansion of the caudal forelimb representation (Kleim et al., 1998; Conner et al., 2003), while our present results indicate that facial nerve transection selectively drives plasticity of neck representations. On the basis of the proposed hypothesis, it was predicted that eliminating the basal forebrain cholinergic system would selectively abolish plasticity of the caudal forelimb representation that requires behavioral activity (skilled forelimb reaching) but would not affect cortical plasticity of the neck representation occurring after facial nerve transection, which does not require behavioral activity.

For this portion of the study, 16 animals received SAP injections into the nucleus basalis and 17 animals received comparable injections of ACSF. Two weeks later, a time period sufficient for elimination of cholinergic projections to the cortex (Conner et al., 2003), animals received bilateral facial nerve transections. After one additional week of recovery, subjects underwent 3 weeks of skilled forelimb motor training (Conner et al., 2003; Whishaw et al., 2003). After completion of skilled forelimb reaching, animals were mapped using standard ICMS techniques. SAP injections in these subjects resulted in elimination of $98 \pm 0.7 \%$ of cortical cholinergic afferent innervation relative to ACSF-injected subjects $(p<0.001)$.

In agreement with previous reports (Conner et al., 2003, 2005), SAP-injected animals exhibited significant deficits in behavioral acquisition on the skilled forelimb reach task, with a $57 \pm 5 \%$ impairment in motor performance across the final $3 \mathrm{~d}$ of testing relative to ACSFinjected controls (Fig. 5; repeated measures ANOVA, $p<0.001$ across all days; $p<0.01$ Student's $t$ test comparing SAP-injected to ACSF-injected subjects on mean performance during last $3 \mathrm{~d}$ of testing; ACSF-injected subjects exhibited $58 \pm 5 \%$ reaching accuracy, and SAP-injected subjects exhibited $25 \pm 5 \%$ ).

Facial motor nerve transections did not alter the ability of animals to acquire the skilled reaching task, nor did it affect the predicted expansion of caudal forelimb representation that occurs following skilled motor training (Fig. 6). Skilled motor training in rats with facial motor nerve transections resulted in a significant expansion of the caudal forelimb representation (6.0 \pm $\left.0.2 \mathrm{~mm}^{2}\right)$ relative to nontrained, facial motor nerve-transected rats $\left(5.2 \pm 0.2 \mathrm{~mm}^{2}\right.$; ANOVA, $p<0.0001$; post hoc TukeyKramer HSD test, $p<0.05)$. Importantly, subjects with specific depletion of cholinergic afferents to the cortex (SAP-injected rats with facial nerve transections) failed to show expansion of the caudal forelimb representation following skilled motor training (caudal forelimb area, $4.8 \pm 0.2 \mathrm{~mm}^{2}$; post hoc Tukey-Kramer HSD, $p<0.001$ compared with trained animals with ACSF injections; $p=0.3$ compared with animals with facial nerve lesions that did not undergo skilled motor training). Thus, cholinergic

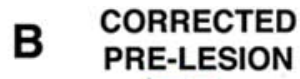

C POST-LESION
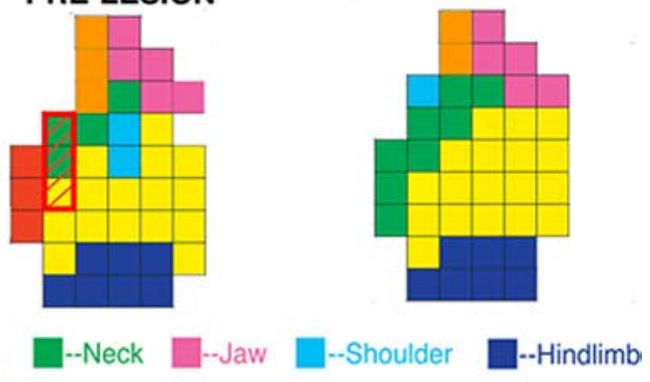

-Neck -Jaw

E

NECK AREA AREA
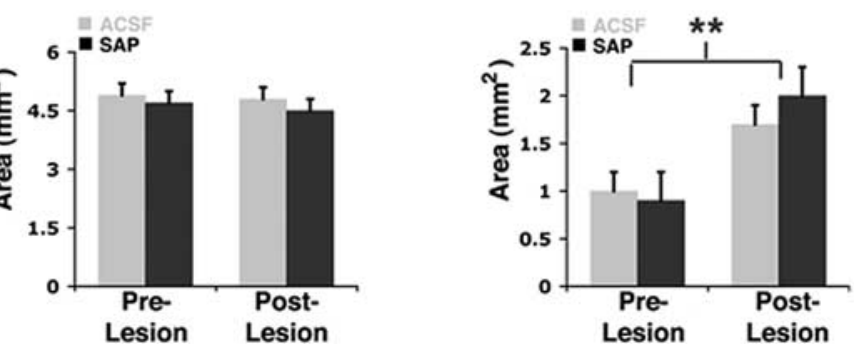

Figure 3. Plasticity following an acute facial motor nerve transection does not require the basal forebrain cholinergic system. A modified ICMS approach was used to characterize cortical plasticity acutely following a facial nerve lesion. $\boldsymbol{A}$, A typical map from 列 a vibrissa region in the same animal, using currents up to $200 \mu \mathrm{A}$, reveals ransection (see Materials and Methods for details). At some vibrissa sites, high amplitude stimulation elicited only vibriss movements, whereas at other vibrissa sites (crosshatch area), additional neck and forelimb movements were evoked at higher stimulus intensities. Calculation of pretransection representational areas was made based on high amplitude stimulation to

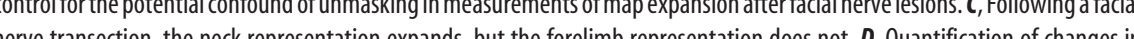
列 the neck representation does not differ between SAP-injected and ACSF-injected subjects ( $p>0.2$ unpaired $t$ tests). Thus, the behaviorally independent plasticity that occurs immediately following a facial nerve transection is not cholinergic-dependent.

ablation completely blocked this behaviorally dependent aspect of cortical plasticity.

However, in the same groups of motor trained subjects, cholinergic depletion had no effect on behaviorally independent motor map plasticity associated with the facial nerve transection. Neck representations were significantly larger in all facial nervetransected groups, both with and without cholinergic depletion, compared with intact animals (Fig. 6; ANOVA, $p<0.01$; TukeyKramer HSD post hoc, $p<0.05$ for all groups of animals relative to pretransection controls). Once again, the extent of neck representation expansion did not differ significantly between SAPinjected and ACSF-injected subjects after facial motor nerve transection ( $p=0.8$, Tukey-Kramer HSD post hoc test).

\section{Discussion}

Results of the present study provide clear evidence that acetylcholine is not essential for all forms of cortical map plasticity in the adult animal. Cholinergic-dependent and cholinergicindependent forms of cortical map plasticity can occur in the same animal and within the same cortical domain, demonstrating that neural mechanisms underlying map plasticity vary according to the experimental paradigm. Moreover, these findings support the hypothesis that cholinergic mechanisms are essential 

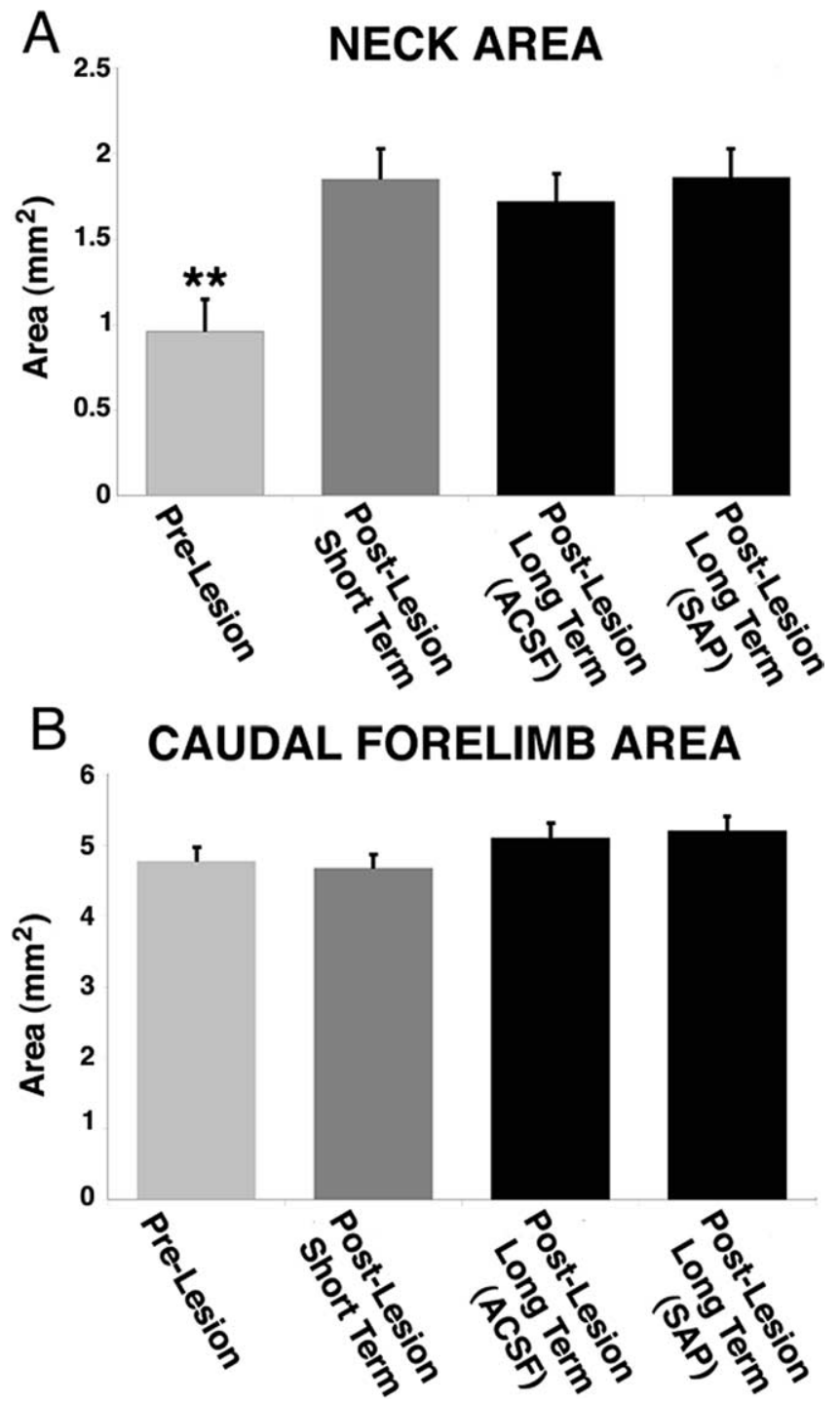

Figure 4. Long-term maintenance of plasticity following a facial motor nerve transection does not require basal forebrain cholinergic mechanisms. $A$, Significant plasticity of neck representations is chronically sustained 6 weeks after facial nerve transection (ANOVA $p<0.01$; all groups differ from pretransection group on Tukey-Kramer post hoc, $p<0.01$ ). Moreover, depleting cholinergic innervation to the cortex does not affect the expression or maintenance of facial nerve transection-induced plasticity: the extent of neck representation plasticity at longterm time points does not differ between SAP-injected and ACSF-injected subjects ( $p=0.8$, post hoc Tukey-Kramer). $\boldsymbol{B}$, As was observed acutely following a facial nerve transection, plasticity of the caudal forelimb representations did not occur at long-term time points (ANOVA, $p>0.05)$.

for plasticity driven by a behaviorally relevant experience (skilled motor training) but are not required for map plasticity that occurs independent of behavioral experience. This study validates previous reports of cholinergic-independent cortical map plasticity (Kamke et al., 2005) and contributes to the development of a theoretical framework for understanding those results. Furthermore, this study demonstrates that the neural mechanisms underlying cortical map reorganization can differ based on the experimental paradigm used to evoke the plasticity.

In the present study, cholinergic lesions blocked plasticity of forelimb motor representations driven by behavioral training but did not block behaviorally independent plasticity of neck motor representations. Although these findings are consistent with the hypothesis that acetylcholine is specifically related to behaviorally

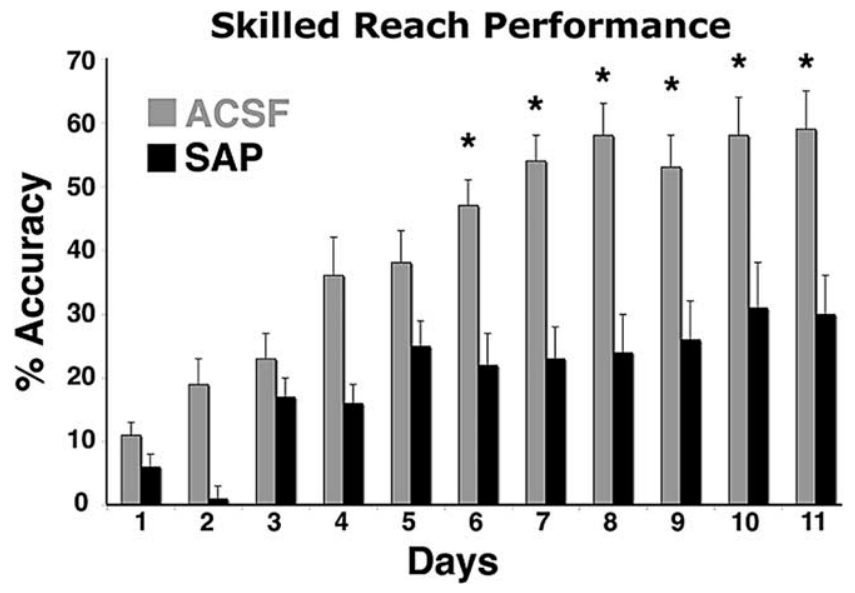

Figure 5. Basal forebrain cholinergic ablation impairs acquisition of skilled forelimb reaching. Ablation of cortical cholinergic innervation results in a significant impairment in acquisition of skilled forelimb grasping (repeated measures ANOVA, $p<0.001$; ${ }^{*}$ significant differences in performance on individual days, $p<0.01$ ). All subjects underwent facial nerve transactions before beginning skilled forelimb reach training.

driven plasticity, it is alternatively possible that the observed plasticity is modality specific, that is, that neck areas of motor cortex can undergo plasticity without cholinergic innervation, while forelimb areas of motor cortex cannot. The latter possibility is unlikely, however. Experimental evidence indicates that motor representations are quite fluid, and a site evoking forelimb movements at one time can evoke neck movements subsequently (Merzenich et al., 1988; Donoghue et al., 1990, 1995; Sanes and Donoghue, 2000). These shifts in representations occur rapidly and are mediated by changes in the balance of excitatory and inhibitory connections that give rise to motor outputs (Jankowska et al., 1975; Jacobs and Donoghue, 1991). Given the fluidity of representations across forelimb and neck regions of motor cortex, it seems unlikely that forelimb motor representations would selectively require acetylcholine for plasticity while neck representations would not. Moreover, afferent cholinergic terminals are distributed similarly across the entire motor cortex, further suggesting acetylcholine is likely to play a comparable role in all areas.

Our present findings substantiate the recent work of Kamke and colleagues (Kamke et al., 2005), demonstrating that cortical map reorganization following peripheral injury can occur independent of cholinergic mechanisms. These results appear to directly contradict earlier studies (Juliano et al., 1991; Webster et al., 1991) reporting that basal forebrain cholinergic mechanisms were required for behaviorally independent somatosensory map plasticity following peripheral nerve lesions. Importantly, techniques used in those earlier studies to ablate the basal forebrain, including electrolytic and excitotoxic lesions, nonselectively damage noncholinergic basal forebrain systems and fibers of passage, including GABAergic, peptidergic, serotonergic, noradrenergic, and dopaminergic projections (Gritti et al., 1997, 2006). Thus, the impact of nonselective basal forebrain lesions on cortical plasticity cannot be attributed specifically to cholinergic substrates. Indeed, previous studies are consistent with the notion that basal forebrain GABAergic substrates are a more likely candidate for modulating rapid cortical reorganization after injury. Pharmacological modulation of intracortical GABAergic mechanisms can mediate shifts in cortical motor representations within minutes (Jacobs and Donoghue, 1991). Moreover, basal forebrain GABAergic neurons selectively innervate cortical 
GABAergic neurons and can directly influence cortical GABAergic mechanisms (Freund and Meskenaite, 1992). These actions are consistent with recent reports implicating the basal forebrain GABAergic corticopetal system in rapidly mediated aspects of higher cognition, such as encoding behavioral salience (Lin and Nicolelis, 2008) and enabling the rapid switching between stimuli and response rules guiding executive functions (Sarter and Bruno, 2002). Use of the 192-IgG-SAP immunotoxin, as was done in the present study and in the study by Kamke and colleagues ( $\mathrm{Ka}$ mke et al., 2005), selectively eliminates only the cholinergic component of afferent basal forebrain innervation to the cortex (Kilgard, 2005), and is therefore a better tool to specifically study the role of the cholinergic system in modulating plasticity.

Previous studies using highly selective ablation of the basal forebrain cholinergic system have also demonstrated that cholinergic mechanisms are essential for mediating sensory plasticity associated with whisker pairing (Baskerville et al., 1997; Sachdev et al., 1998; Zhu and Waite, 1998). Selective blockade of cholinergic signaling within the barrel cortex similarly impairs whisker pairing plasticity (Maalouf et al., 1998). Although the contribution of behavioral experience in these studies of somatosensory plasticity was not specifically controlled for, recent evidence strongly suggests that sensory stimulation is only effective at inducing plasticity in the context of sensory-guided behavioral learning (Blake et al., 2006), requiring attentional mechanisms (Recanzone et al., 1992). Sensory stimulation in the absence of attention and behavioral engagement fails to induce plasticity of cortical representations (Recanzone et al., 1992; Blake et al., 2006). Thus, it is likely that plasticity associated with whisker pairing may require cholinergic mechanisms that are engaged in the context of increased attentional demand.

It is not completely understood why cholinergic input would only be required for cortical plasticity driven by behavioral paradigms. However, it may be postulated that the basal forebrain cholinergic system influences cortical plasticity during behavioral training specifically through its role in modulating attentional mechanisms. Indeed, previous studies have indicated that the basal forebrain cholinergic system becomes most relevant in tasks in which attentional load is increased (McGaughy et al., 1996; Turchi and Sarter, 1997). The cholinergic system may be necessary for the normal allocation of attention to specific cortical domains or may enable cortical activity arising from afferent sensory inputs during a relevant learning experience to drive plasticity of cortical representations $(\mathrm{Gu}, 2002)$. Additional work will be required to delineate the exact role this system plays in modulating plasticity.

Acetylcholine, with other neuromodulatory systems, has previously been reported to exert a role in modulating ocular dominance plasticity, a form of plasticity that occurs in the developing visual cortex (Kasamatsu and Pettigrew, 1976; Hubel et al., 1977; Daw et al., 1983; Shirokawa et al., 1989; Gu and Singer, 1993,
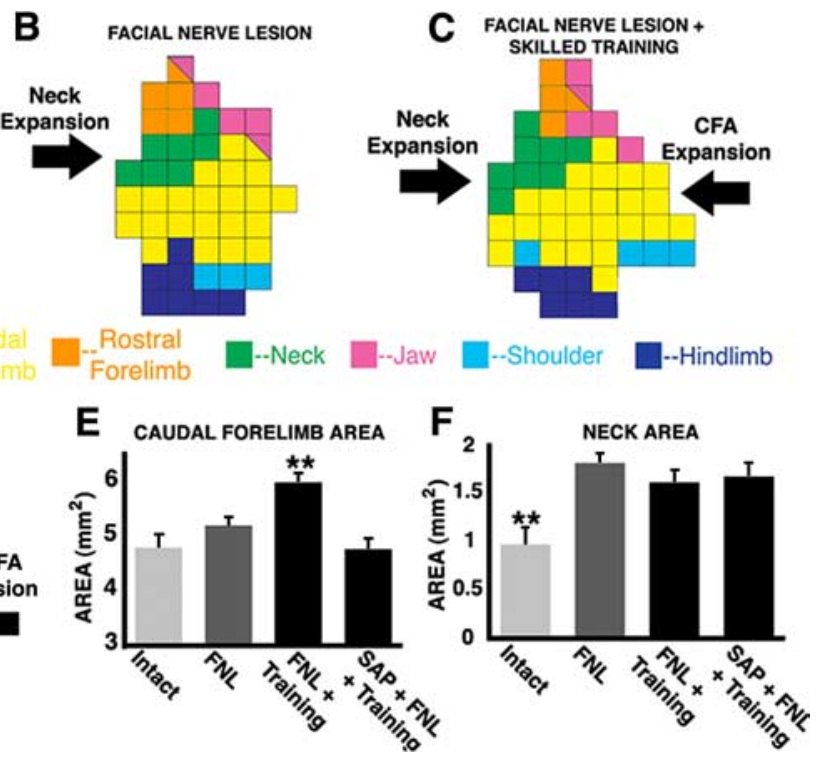

Figure 6. Cholinergic ablation selectively abolishes plasticity associated with behavioral experience. $\boldsymbol{A}-\boldsymbol{D}$, Representative motor maps of different animals from each experimental group (intact animal, long-term postfacial nerve lesion, and animals , $F$, Quation of map plasticity across all experimental subjects. $\boldsymbol{E}$, Skilled motor training results in significant expansion of independent plasticity of neck representations induced by a facial nerve transection (ANOVA, $p<0.01 ; p<0.05$, Tukey-Kramer HSD post hoc comparing intact to all facial nerve transected subjects. No other significant differences were seen.).

1995). Ablation of either cholinergic or noradrenergic systems alone in developing visual cortex was not sufficient, however, to completely block ocular dominance plasticity; instead, both cholinergic and noradrenergic systems must be abolished concurrently to block this form of plasticity (Bear and Singer, 1986). In contrast, we find that isolated elimination of cholinergic systems abolishes behaviorally dependent motor cortex plasticity in the adult brain. Although cholinergic lesions alone do not abolish behaviorally independent plasticity associated with facial nerve transection in the adult, it is possible that abolishing multiple neuromodulatory systems (such as cholinergic along with noradrenergic and/or serotonergic systems) would abolish such plasticity. Additional studies are necessary to understand how multiple neuromodulatory systems contribute to distinct forms of sensory and motor plasticity.

Previous studies have documented various aspects of neuronal plasticity associated with chronic peripheral nerve damage in conjunction with the reorganization of cortical sensory and motor representations. Over very long time periods (years), peripheral lesions result in dramatic reorganization of dendritic and axonal arbors at both cortical and subcortical levels in somatosensory regions (Florence and Kaas, 1995; Florence et al., 1998). Although the present findings, and those of others (Kamke et al., 2005), indicate that cortical map reorganization following peripheral damage occurs without behavioral involvement and independent of cholinergic mechanisms, it remains to be determined whether long-term changes in anatomical plasticity require behavioral activity or a cholinergic substrate. Evidence indicating that phantom limb pain following limb amputation may be related to aberrant plasticity (Flor et al., 1995) emphasizes 
the importance of elucidating mechanisms of plasticity following peripheral nerve injury.

The present study suggests that mechanisms underlying motor cortex plasticity following peripheral nerve injury differ from plasticity mechanisms engaged by behavioral experience. Interestingly, CNS injuries have been associated with both automatic, nonbehaviorally mediated forms of plasticity, as well as plasticity driven specifically by behavioral interventions such as motor rehabilitation (Nudo et al., 1996a; Dijkhuizen et al., 2001; Dijkhuizen et al., 2003; Frost et al., 2003; Dobkin, 2004; Ramanathan et al., 2006). On the basis of the present results, it is hypothesized that cholinergic mechanisms would be required for aspects of plasticity that are behaviorally dependent but not for those that are behaviorally independent. Previous studies investigating the role of basal forebrain cholinergic mechanisms in modulating plasticity following central lesions have indeed demonstrated that specific rehabilitative motor training of the forelimb following a focal cortical injury resulted in cortical plasticity only in the presence of an intact basal forebrain cholinergic system (Conner et al., 2005). More importantly, functional recovery following focal cortical injury was associated specifically with the behaviorally driven and cholinergic-dependent aspects of plasticity (Conner et al., 2005). Together, these past and present findings support the hypothesis that cholinergic mechanisms are a critical component for enabling behaviorally driven plasticity of cortical circuits and raise the possibility that rehabilitative strategies that specifically evoke greater attentional demand, and subsequently recruit cholinergic mechanisms, may promote cortical plasticity and improve functional outcomes.

Although most studies have implicated the basal forebrain cholinergic system in mediating the reorganization of motor, sensory, or auditory cortical maps, such plasticity can occur in the absence of cholinergic mechanisms under certain circumstances (Kamke et al., 2005). To date, no unifying concept has emerged to account for differing demands for cholinergic systems to achieve cortical plasticity in various experimental paradigms. The present study presents a new theoretical framework for the involvement of basal forebrain cholinergic mechanisms in cortical plasticity within adult animals, indicating that cholinergic dependency of cortical plasticity varies as a function of the behavioral demands of a task. Using experimental paradigms to evoke distinct forms of plasticity in the same brain region, and within the same animal, we demonstrate a unique dependency for cholinergic inputs only under conditions in which plasticity is driven by behavior. These novel findings reconcile apparent discrepancies in the field and establish a unifying model in which basal forebrain cholinergic mechanisms are required specifically for behaviorally mediated facilitation of cortical map plasticity.

\section{References}

Bakin JS, Weinberger NM (1996) Induction of a physiological memory in the cerebral cortex by stimulation of the nucleus basalis. Proc Natl Acad Sci U S A 93:11219-11224.

Bakin JS, South DA, Weinberger NM (1996) Induction of receptive field plasticity in the auditory cortex of the guinea pig during instrumental avoidance conditioning. Behav Neurosci 110:905-913.

Baskerville KA, Schweitzer JB, Herron P (1997) Effects of cholinergic depletion on experience-dependent plascticity in the cortex of the rat. Neuroscience 80:1159-1169.

Baxter MG, Bucci DJ, Gorman LK, Wiley RG, Gallagher M (1995) Selective immunotoxic lesions of basal forebrain cholinergic cells: effects on learning and memory in rats. Behav Neurosci 109:714-722.

Bear MF, Singer W (1986) Modulation of visual cortical plasticity by acetylcholine and noradrenaline. Nature 320:172-176.

Blake DT, Heiser MA, Caywood M, Merzenich MM (2006) Experience- dependent adult cortical plasticity requires cognitive association between sensation and reward. Neuron 52:371-381.

Book AA, Wiley RG, Schweitzer JB (1992) Specificity of 192 IgG-saporin for NGF receptor-positive cholinergic basal forebrain neurons in the rat. Brain Res 590:350-355.

Butt AE, Testylier G, Dykes RW (1997) Acetylcholine release in rat frontal and somatosensory cortex is enhanced during tactile discrimination learning. Psychobiology 25:18-33.

Conner JM, Darracq MA, Roberts J, Tuszynski MH (2001) Nontropic actions of neurotrophins: subcortical nerve growth factor gene delivery reverses age-related degeneration of primate cortical cholinergic innervation. Proc Natl Acad Sci U S A 98:1941-1946.

Conner JM, Culberson A, Packowski C, Chiba AA, Tuszynski MH (2003) Lesions of the basal forebrain cholinergic system impair task acquisition and abolish cortical plasticity associated with motor skill learning. Neuron 38:819-829.

Conner JM, Chiba AA, Tuszynski MH (2005) The basal forebrain cholinergic system is essential for cortical plasticity and functional recovery following brain injury. Neuron 46:173-179.

Daw NW, Rader RK, Robertson TW, Ariel M (1983) Effects of 6-hydroxydopamine on visual deprivation in the kitten striate cortex. J Neurosci 3:907-914.

Dijkhuizen RM, Ren J, Mandeville JB, Wu O, Ozdag FM, Moskowitz MA, Rosen BR, Finklestein SP (2001) Functional magnetic resonance imaging of reorganization in rat brain after stroke. Proc Natl Acad Sci U S A 98:12766-12771.

Dijkhuizen RM, Singhal AB, Mandeville JB, Wu O, Halpern EF, Finklestein SP, Rosen BR, Lo EH (2003) Correlation between brain reorganization, ischemic damage, and neurologic status after transient focal cerebral ischemia in rats: a functional magnetic resonance imaging study. J Neurosci 23:510-517.

Di Patre PL, Mathes CW, Butcher LL (1993) Differential visualization of cholinesterasic neuronal somata and fibers by use of modifications of acetylcholinesterase pharmacohistochemistry. J Histochem Cytochem 41:129-135.

Dobkin BH (2004) Neurobiology of rehabilitation. Ann N Y Acad Sci 1038:148-170.

Donoghue JP (1995) Plasticity of adult sensorimotor representations. Curr Opin Neurobiol 5:749-754.

Donoghue JP, Suner S, Sanes JN (1990) Dynamic organization of primary motor cortex output to target muscles in adult rats. II. Rapid reorganization following motor nerve lesions. Exp Brain Res 79:492-503.

Flor H, Elbert T, Knecht S, Wienbruch C, Pantev C, Birbaumer N, Larbig W, Taub E (1995) Phantom-limb pain as a perceptual correlate of cortical reorganization following arm amputation. Nature 375:482-484.

Florence SL, Kaas JH (1995) Large-scale reorganization at multiple levels of the somatosensory pathway follows therapeutic amputation of the hand in monkeys. J Neurosci 15:8083-8095.

Florence SL, Taub HB, Kaas JH (1998) Large-scale sprouting of cortical connections after peripheral injury in adult macaque monkeys. Science 282:1117-1121.

Freund TF, Meskenaite V (1992) $\gamma$-Aminobutyric acid-containing basal forebrain neurons innervate inhibitory interneurons in the neocortex. Proc Natl Acad Sci U S A 89:738-742.

Frost SB, Barbay S, Friel KM, Plautz EJ, Nudo RJ (2003) Reorganization of remote cortical regions after ischemic brain injury: a potential substrate for stroke recovery. J Neurophysiol 89:3205-3214.

Geula C, Mesulam MM (1996) Systematic regional variations in the loss of cortical cholinergic fibers in Alzheimer's disease. Cereb Cortex 6:165-177.

Gritti I, Mainville L, Mancia M, Jones BE (1997) GABAergic and other noncholinergic basal forebrain neurons, together with cholinergic neurons, project to the mesocortex and isocortex in the rat. J Comp Neurol 383:163-177.

Gritti I, Henny P, Galloni F, Mainville L, Mariotti M, Jones BE (2006) Stereological estimates of the basal forebrain cell population in the rat, including neurons containing choline acetyltransferase, glutamic acid decarboxylase or phosphate-activated glutaminase and colocalizing vesicular glutamate transporters. Neuroscience 143:1051-1064.

Gu Q (2002) Neuromodulatory transmitter systems in the cortex and their role in cortical plasticity. Neuroscience 111:815-835.

Gu Q, Singer W (1993) Effects of intracortical infusion of anticholinergic 
drugs on neuronal plasticity in kitten striate cortex. Eur J Neurosci 5:475-485.

Gu Q, Singer W (1995) Involvement of serotonin in developmental plasticity of kitten visual cortex. Eur J Neurosci 7:1146-1153.

Heckers S, Ohtake T, Wiley RG, Lappi DA, Geula C, Mesulam MM (1994) Complete and selective cholinergic denervation of rat neocortex and hippocampus but not amygdala by an immunotoxin against the p75 NGF receptor. J Neurosci 14:1271-1289.

Hubel DH, Wiesel TN, LeVay S (1977) Plasticity of ocular dominance columns in monkey striate cortex. Philos Trans R Soc Lond B Biol Sci 278:377-409.

Jacobs KM, Donoghue JP (1991) Reshaping the cortical motor map by unmasking latent intracortical connections. Science 251:944-947.

Jankowska E, Padel Y, Tanaka R (1975) The mode of activation of pyramidal tract cells by intracortical stimuli. J Physiol 249:617-636.

Juliano SL, Ma W, Eslin D (1991) Cholinergic depletion prevents expansion of topographic maps in somatosensory cortex. Proc Natl Acad Sci U S A 88:780-784.

Kamke MR, Brown M, Irvine DR (2005) Basal forebrain cholinergic input is not essential for lesion-induced plasticity in mature auditory cortex. Neuron 48:675-686.

Kasamatsu T, Pettigrew JD (1976) Depletion of brain catecholamines: failure of ocular dominance shift after monocular occlusion in kittens. Science 194:206-209.

Kilgard MP (2005) Cortical map reorganization without cholinergic modulation. Neuron 48:529-530.

Kilgard MP, Merzenich MM (1998) Cortical map reorganization enabled by nucleus basalis activity. Science 279:1714-1718.

Kleim JA, Barbay S, Nudo RJ (1998) Functional reorganization of the rat motor cortex following motor skill learning. J Neurophysiol 80: $3321-3325$.

Liepert J, Miltner WH, Bauder H, Sommer M, Dettmers C, Taub E, Weiller C (1998) Motor cortex plasticity during constraint-induced movement therapy in stroke patients. Neurosci Lett 250:5-8.

Lin SC, Nicolelis MA (2008) Neuronal ensemble bursting in the basal forebrain encodes salience irrespective of valence. Neuron 59:138-149.

Ma X, Suga N (2005) Long-term cortical plasticity evoked by electric stimulation and acetylcholine applied to the auditory cortex. Proc Natl Acad Sci U S A 102:9335-9340.

Maalouf M, Miasnikov AA, Dykes RW (1998) Blockade of cholinergic receptors in rat barrel cortex prevents long-term changes in the evoked potential during sensory preconditioning. J Neurophysiol 80:529-545.

McGaughy J, Kaiser T, Sarter M (1996) Behavioral vigilance following infusions of 192 IgG-saporin into the basal forebrain: selectivity of the behavioral impairment and relation to cortical AChE-positive fiber density. Behav Neurosci 110:247-265.

McLin DE 3rd, Miasnikov AA, Weinberger NM (2002) Induction of behavioral associative memory by stimulation of the nucleus basalis. Proc Natl Acad Sci U S A 99:4002-4007.

Merzenich MM, Recanzone G, Jenkins WM, Allard TT, Nudo RT (1988) Cortical representational plasticity. In: Neurobiology of neocortex (Rakic P, Singer W, eds), pp 41-67. New York: Wiley.

Nelles G (2004) Cortical reorganization-effects of intensive therapy. Restor Neurol Neurosci 22:239-244.

Nudo RJ, Milliken GW (1996) Reorganization of movement representations in primary motor cortex following focal ischemic infarcts in adult squirrel monkeys. J Neurophysiol 75:2144-2149.

Nudo RJ, Wise BM, SiFuentes F, Milliken GW (1996a) Neural substrates for the effects of rehabilitative training on motor recovery after ischemic infarct [see comments]. Science 272:1791-1794.

Nudo RJ, Milliken GW, Jenkins WM, Merzenich MM (1996b) Usedependent alterations of movement representations in primary motor cortex of adult squirrel monkeys. J Neurosci 16:785-807.

Pang KC, Nocera R, Secor AJ, Yoder RM (2001) GABAergic septohippocampal neurons are not necessary for spatial memory. Hippocampus $11: 814-827$.
Prakash N, Cohen-Cory S, Penschuck S, Frostig RD (2004) Basal forebrain cholinergic system is involved in rapid nerve growth factor (NGF)induced plasticity in the barrel cortex of adult rats. J Neurophysiol 91:424-437.

Ramanathan D, Conner JM, Tuszynski MH (2006) A form of motor cortical plasticity that correlates with recovery of function after brain injury. Proc Natl Acad Sci U S A 103:11370-11375.

Recanzone GH, Merzenich MM, Jenkins WM, Grajski KA, Dinse HR (1992) Topographic reorganization of the hand representation in cortical area $3 \mathrm{~b}$ owl monkeys trained in a frequency-discrimination task. J Neurophysiol 67:1031-1056.

Richardson RT, DeLong MR (1991) Electrophysiological studies of the functions of the nucleus basalis in primates. Adv Exp Med Biol 295:233-252.

Sachdev RNS, Lu S-M, Wiley RG, Ebner FF (1998) Role of basal forebrain cholinergic projection in somatosensory cortical plasticity. J Neurophysiol 79:3216-3228.

Sanes JN, Donoghue JP (2000) Plasticity and primary motor cortex. Annu Rev Neurosci 23:393-415.

Sanes JN, Suner S, Lando JF, Donoghue JP (1988) Rapid reorganization of adult rat motor cortex somatic representation patterns after motor nerve injury. Proc Natl Acad Sci U S A 85:2003-2007.

Sanes JN, Suner S, Donoghue JP (1990) Dynamic organization of primary motor cortex output to target muscles in adult rats. I. Long-term patterns of reorganization following motor or mixed peripheral nerve lesions. Exp Brain Res 79:479-491.

Sarter M, Bruno JP (1997) Cognitive functions of cortical acetylcholine: toward a unifying hypothesis. Brain Res Brain Res Rev 23:28-46.

Sarter M, Bruno JP (2002) The neglected constituent of the basal forebrain corticopetal projection system: GABAergic projections. Eur J Neurosci 15:1867-1873.

Sarter M, Hasselmo ME, Bruno JP, Givens B (2005) Unraveling the attentional functions of cortical cholinergic inputs: interactions between signal-driven and cognitive modulation of signal detection. Brain Res Brain Res Rev 48:98-111.

Semba K, Egger MD (1986) The facial "motor" nerve of the rat: control of vibrissal movement and examination of motor and sensory components. J Comp Neurol 247:144-158.

Shirokawa T, Kasamatsu T, Kuppermann BD, Ramachandran VS (1989) Noradrenergic control of ocular dominance plasticity in the visual cortex of dark-reared cats. Brain Res Dev Brain Res 47:303-308.

Turchi J, Sarter M (1997) Cortical acetylcholine and processing capacity: effects of cortical cholinergic deafferentation on crossmodal divided attention in rats. Brain Res Cogn Brain Res 6:147-158.

VandenBerg PM, Hogg TM, Kleim JA, Whishaw IQ (2002) Long-Evans rats have a larger cortical topographic representation of movement than Fischer-344 rats: a microstimulation study of motor cortex in naive and skilled reaching-trained rats. Brain Res Bull 59:197-203.

Webster HH, Hanisch UK, Dykes RW, Biesold D (1991) Basal forebrain lesions with or withour reserpine injection inhibit cortical reorganization in rat hindpaw primary somatosensory cortex following sciatic nerve section. Somatosens Mot Res 8:327-346.

Weinberger NM (2003) The nucleus basalis and memory codes: auditory cortical plasticity and the induction of specific, associative behavioral memory. Neurobiol Learn Mem 80:268-284.

Whishaw IQ, Gorny B, Foroud A, Kleim JA (2003) Long-Evans and Sprague-Dawley rats have similar skilled reaching success and limb representations in motor cortex but different movements: some cautionary insights into the selection of rat strains for neurobiological motor research. Behav Brain Res 145:221-232.

Wilson FAW, Rolls ET (1990) Learning and memory is reflected in the response of reinforcement-related neurons in the primate basal forebrain. J Neurosci 10:1254-1267.

Zhu XO, Waite PM (1998) Cholinergic depletion reduces plasticity of barrel field cortex. Cereb Cortex 8:63-72.

Zilles K (1985) The cortex of the rat: a stereotaxic atlas. Berlin: Springer. 